\title{
On the boundaries of a convective zone and the extent of overshooting
}

\author{
L. Deng ${ }^{1}$ and D.R. Xiong ${ }^{2}$ \\ ${ }^{1}$ National Astronomical Observatories, Chinese Academy of Sciences, Beijing 100012 \\ licai@bao.ac.cn \\ ${ }^{2}$ Purple Mountain Observatory, Chinese Academy of Sciences, Nanjing 210008 \\ Xiongdr@pmo.ac.cn
}

\begin{abstract}
In this work, we will show that a proper definition of the boundary of a convective zone should be the place where the convective energy flux (i.e. the correlation of turbulent velocity and temperature) changes its sign. Therefore, it is convectively unstable region when the flux is positive, and it is convective overshooting zone when the flux becomes negative. In our nonlocal convection theory, convection is already sub-adiabatic $\left(\nabla<\nabla_{a d}\right)$ far before reaching the unstable boundary; while in the overshooting zone below the convective zone, convection is sub-adiabatic and super-radiative $\left(\nabla_{r a d}<\nabla<\nabla_{a d}\right)$. The transition between the adiabatic temperature gradient and the radiative one is continuous and smooth instead of a sudden switch. In the unstable zone, the temperature gradient is approaching radiative rather than going to adiabatic. The distance of convective overshooting is different for different physical quantities. The overshooting distance in the context of stellar evolution, measured by the extent of mixing of stellar matter, should be more extended than that of other physical quantities. It is estimated as large as $0.25-1.7 \mathrm{H}_{p}$ depending on the evolutionary timescale.
\end{abstract}

Keywords. convection, stars:evolution

\section{Introduction}

As the classical treatment of convection, the local theory has been used in modelling stellar structure and evolution. In the calculation of massive star evolution, Schwarzschild \& Härm (1958) discovered that the hydrogen rich radiative envelope just outside the helium rich convective core cannot be convectively stable, and that leaded to the paradox of so called semi-convection. To solve that problem, the idea of semi-convection was initiated, i.e. the region outside the convective core is in a state of semi-convection. Stellar matter in this region is nearly in neutral stability $\left(\nabla \leqslant \nabla_{a d}\right)$, therefore convective energy transport due to this mild convection can be neglected, while the mixing of chemical compositions should be important, which makes a gradient of molecular weight in this region (otherwise called semi-convection zone). There had been a great debate in the community for a long period since then on whether the Schwarzschild or Ledoux criteria should be applied for the neutral stability of convection, and whether the semi-convective zone should be very wide or rather narrow. Stothers (1970) commented on various establishments of semi-convection. Evolutionary scenarios for massive stars with or without semi-convection were also discussed (e.g. Chiosi \& Summa 1970). It has been realized later that the problem of semi-convection is in fact due to the non-locality of stellar convection. Therefore various theories of non-local theory of stellar convection have been worked out (Spiegel 1963, Ulrich 1970, Xiong 1977, 1981, 1989, Kufuss 1986, Grossman et al. 1993, Canuto 1993, Canuto \& Dubovikov 1998). Generally speaking, non-local theory of convection makes the results better match observations than the local ones. 

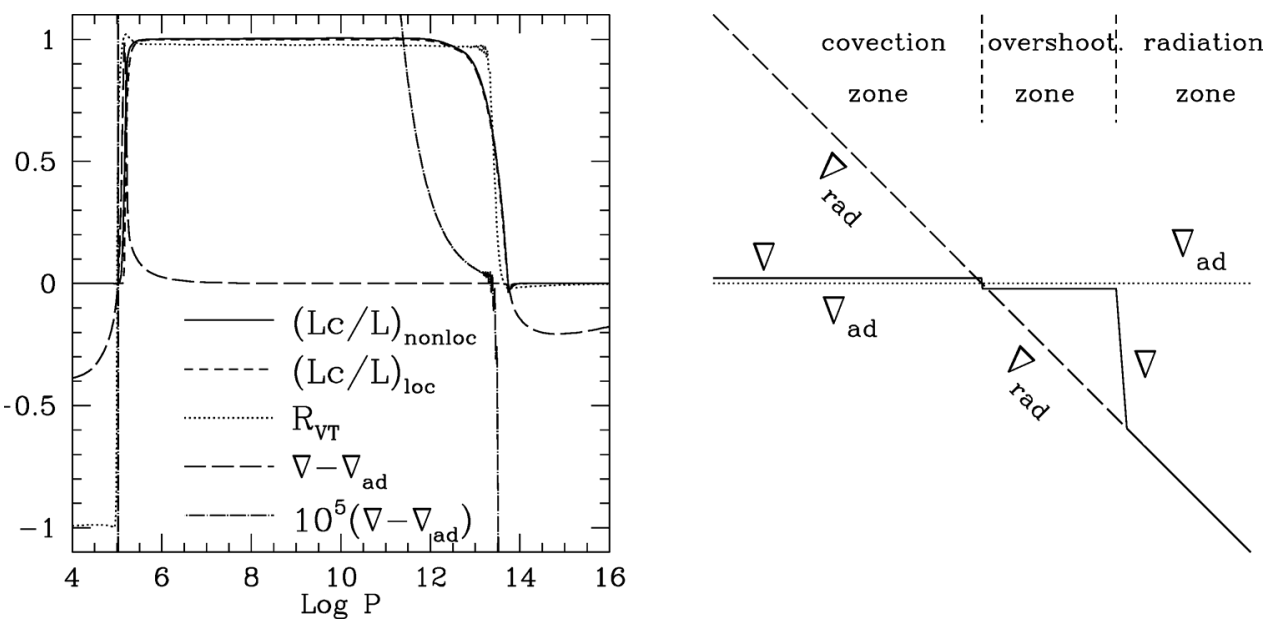

Figure 1. The left panel: the super-adiabatic temperature gradient $\nabla-\nabla_{a d}$, turbulent velocity-temperature correlation $R_{V T}$, and the fractional convective flux $L_{c} / L$ versus the depth $(\log P)$ for a non-local convection model of the Sun. The dashed line is the fractional convective flux $L_{c} / L$ for a local model with the same depth of convective zone. The right panel: A sketch of the lower convective and overshooting zones in the usual phenomenological non-local mixing length theory (Monteiro et al. 2000).

However, the non-local theory of convection is rather complicated, which is much less straightforward to be understood, much more difficult to be applied and much more computing power demanding than the phenomenological local (Böhm-Vitense 1958) and non-local mixing length theories (Maeder 1975, Bressan et al. 1981). In this work, we will address the issues of the boundary of a convective zone and the related problems of convective overshoot.

\section{How to define the boundaries of a convective zone?}

Normally in a local theory of convection, the boundary of convective zone is given by the so called Schwarzschild's criterion, $\nabla=\nabla_{a d}$,

and for chemical inhomogeneous media, the Ledoux criterion applies, $\nabla=\nabla_{a d}+\nabla_{\mu}$, which is derived by analysis of the local convective stability.

Based on our nonlocal statistic theory of correlation functions, the boundary of the (non-local) convective zone is set at where the turbulent velocity-temperature correlation $V$ vanishes (for definition of the quantities, see our previous work, e.g. Deng \& Xiong [2008]),

$$
V=0
$$

Passing through the boundary, $V$ changes its sign: within the convective zone:

$$
V>0
$$

and in the overshooting zone:

$$
V<0 \text {. }
$$

It is clear from the left panel in Fig. 1 that if the boundary is defined as such, the structures of the local and non-local convection models with the same depth of convective 
zone should be similar. It can be understood by the fact that, within the stellar interior, the turbulent kinetic energy flux $\left(L_{t}\right)$ is generally much less than that of thermal convection $\left(L_{c}\right)$, and the turbulent pressure $\left(P_{t}=\rho x^{2}\right)$ is much less than that of gas $\left(P_{g}\right)$. The thermal structure derived from our non-local convection theory is different from that from usual non-local mixing length theory as sketched in the right panel of Fig. 1.

The left panel in Fig. 1 clearly demonstrates that convective motions near the upper and lower boundaries of the convective zone are very different. This is due to the fact that, in the atmosphere, the density is very low and $P_{e}=x / x_{c}<1$, therefore convective energy transfer is inefficient. As a result, there exists a thin super-adiabatic layer atop of the convective zone. Passing through the upper boundary, the turbulent velocity-temperature correlation $R_{V T}=V / x Z^{1 / 2}$ drops quickly from $\sim 1$ to $\sim-1$. This theoretical prediction agrees the observations of the solar granular velocity field (Leighton et al. 1962; Salucci et al. 1994) and the results of hydrodynamic simulations (Kupka 2003). Contrary to the situations in the solar atmosphere, convection is highly efficient in terms of energy transfer $\left(P_{e} \gg 1\right)$ in the deep interiors of the Sun. Towards the lower boundary of the convective zone, the turbulent velocity-temperature correlation $R_{V T}$ decreases abruptly and approaches zero $\left(\left|R_{V T}\right| \ll 1\right)$. What makes it so different at the two boundaries is the distinct the effective Peclet number (see Deng \& Xiong 2008 for details).

\section{How extended is convective overshooting?}

Due to the complexity of non-local convection theory, almost all the modellings of stellar structure and evolution are still using the local convection theory. Convective overshooting is defined as the penetration of convective motion through the classical boundary of convectively unstable zone into the adjacent stable region. The extent of overshooting is not the same for different physical quantities following our dynamic theory of convection, and this leads to some troubles in understanding the true nature and estimation the extension of overshooting.

The overshooting distance defined by dropping off of Lithium abundance by a factor of $e$ is shown in the 8th column of table 1, while that defined by the distance from the boundary of convective zone to where Lithium becomes zero is given in the last column of table 1 . It is clearly from figs. $2 \mathrm{a}-\mathrm{d}$ that Lithium abundance vanishes very quickly after the e-folding depletion, the distance between them is less than $0.1 \mathrm{H}_{P}$. Completely different from the Lithium abundance profile in fig. 2 , turbulent velocity $(x)$, temperature fluctuation $\left(Z^{1 / 2}\right)$ and velocity-temperature correlation $(V)$ decrease exponentially with depth in the overshooting zone. The e-folding distances determined from the curves are given in the 2 nd -4 th colums (for the upper part of overshooting zone), and 4 th- 7 th columns (the lower part of overshooting zone). The e-folding distances given by turbulent velocity and temperature fields are very close to the analytic asymptote in our theory (Xiong 1989b). They hardly change with stellar mass, and are rather different from the e-folding distances defined by Lithium depletion.

The overshooting distance that is important to stellar evolution is the extension of the non-local convective mixing of chemical elements. Obviously, it is neither that of convective energy transfer nor those of turbulent velocity and temperature fluctuations. Calculations of massive star evolution under our complete non-local theory of convection shown that the non-local convective mixing overshoots a very extended distance (Xiong 1986).

Although the overshooting at the bottom of the solar convective zone cannot be observed directly, we fortunately have another excellent indicator for the extension of overshooting, which is the Lithium abundance of the Sun and solar type stars. 
Table 1. The e-folding lengths measured in local pressure scale height.

\begin{tabular}{cccccccccc}
\hline \multicolumn{8}{c}{ upper oversh. zone } & \multicolumn{1}{c}{ lower oversh. zone } \\
$\mathrm{M} / \mathrm{M}_{\odot}$ & $\mathrm{x}$ & $\mathrm{z}$ & $\mathrm{V}$ & $\mathrm{x}$ & $\mathrm{z}$ & $\mathrm{V}$ & $\mathrm{Li}$ & Dcut \\
\hline 0.800 & 0.47 & 0.36 & 0.20 & 0.25 & 0.25 & 0.080 & 0.26 & 0.30 \\
0.850 & 0.48 & 0.36 & 0.21 & 0.25 & 0.25 & 0.081 & 0.36 & 0.38 \\
0.900 & 0.50 & 0.35 & 0.20 & 0.25 & 0.25 & 0.082 & 0.42 & 0.54 \\
0.925 & 0.50 & 0.36 & 0.21 & 0.25 & 0.25 & 0.069 & 0.50 & 0.58 \\
0.950 & 0.50 & 0.33 & 0.20 & 0.26 & 0.25 & 0.076 & 0.60 & 0.69 \\
0.975 & 0.49 & 0.33 & 0.20 & 0.31 & 0.31 & 0.104 & 0.67 & 0.74 \\
1.000 & 0.50 & 0.32 & 0.20 & 0.29 & 0.31 & 0.093 & 0.85 & 0.91 \\
1.025 & 0.63 & 0.30 & 0.21 & 0.29 & 0.29 & 0.096 & 1.05 & 1.11 \\
1.050 & 0.52 & 0.30 & 0.19 & 0.30 & 0.36 & 0.092 & 1.25 & 1.33 \\
1.075 & 0.60 & 0.30 & 0.21 & 0.38 & 0.38 & 0.114 & 1.64 & 1.69 \\
\hline
\end{tabular}

Figs. 2a-2d show the Lithium abundance depletions due to overshooting for 4 given mass (indicated in the corresponding panels) stellar models as functions of depth $(\log P)$, when the effect of evolution is not considered. The dashed lines in the plots indicate the boundary of convective zone. As from fig. 2c, there is a gradually accelerating reduction of Lithium abundance in the overshooting zone. In the upper part of the overshooting zone, the mixing caused by non-local convection is very efficient, the abundance keeps the same as in the convectively unstable zone for about $0.4 \mathrm{H}_{P}$ in length downwards. It is then followed by a partially mixed region where Lithium abundance is reduced quicker toward the center and vanishes suddenly, such a partial mixing region is about $0.5 \mathrm{H}_{P}$ in depth. Even deeper is the non-mixing zone. If the overshooting distance is taken as the e-folding length of the abundance from the bottom of the convective zone, it reads about $0.83 \mathrm{H}_{P}$; otherwise if we count the deeppest bottom of the mixing process, it reads about $0.9 \mathrm{H}_{P}$ for the solar model.

\section{Summary and discussions}

Detailed discussions on the definition of the boundary of convective zone, and the distance of convective overshooting are presented in this paper. The main results can be summarized in the following:

(a) Choosing the place where the convective flux (or equivalently the turbulent velocity-temperature correlation) changes it sign as the boundary is the most proper and convenient, where the convective flux is greater than zero is convectively unstable zone, while it is the overshooting zone when the convective flux is smaller than zero;

(b) It is not quite right to talk about a general overshooting distance for stellar convection. The distance of overshooting is different for different physical quantities. The overshooting distances of turbulent velocity and temperature fluctuations are quite extended, the e-folding lengths can reach $0.25-0.5 \mathrm{H}_{P}$. Very extended and weak overshooting can still induce fairly efficient mixing in a very long timescale of evolution. Therefore we anticipate a very extended overshooting for mixing of matters, the e-folding lengths of which is generally larger than that of turbulent velocity and temperature. The one in massive stellar model, for instance, can reach 1 pressure scale height (Xiong 1986).

Using a similar non-local convection theory, we have calculated the early stages of the post-main-sequence evolution for massive stars. The overshooting distances from the cores are rather extended, being typically $1-2 H_{P}$, the fractional mass within the overshooting zone $M_{\text {over }} / M_{0} \sim 0.1--0.5$ (Xiong 1986). 

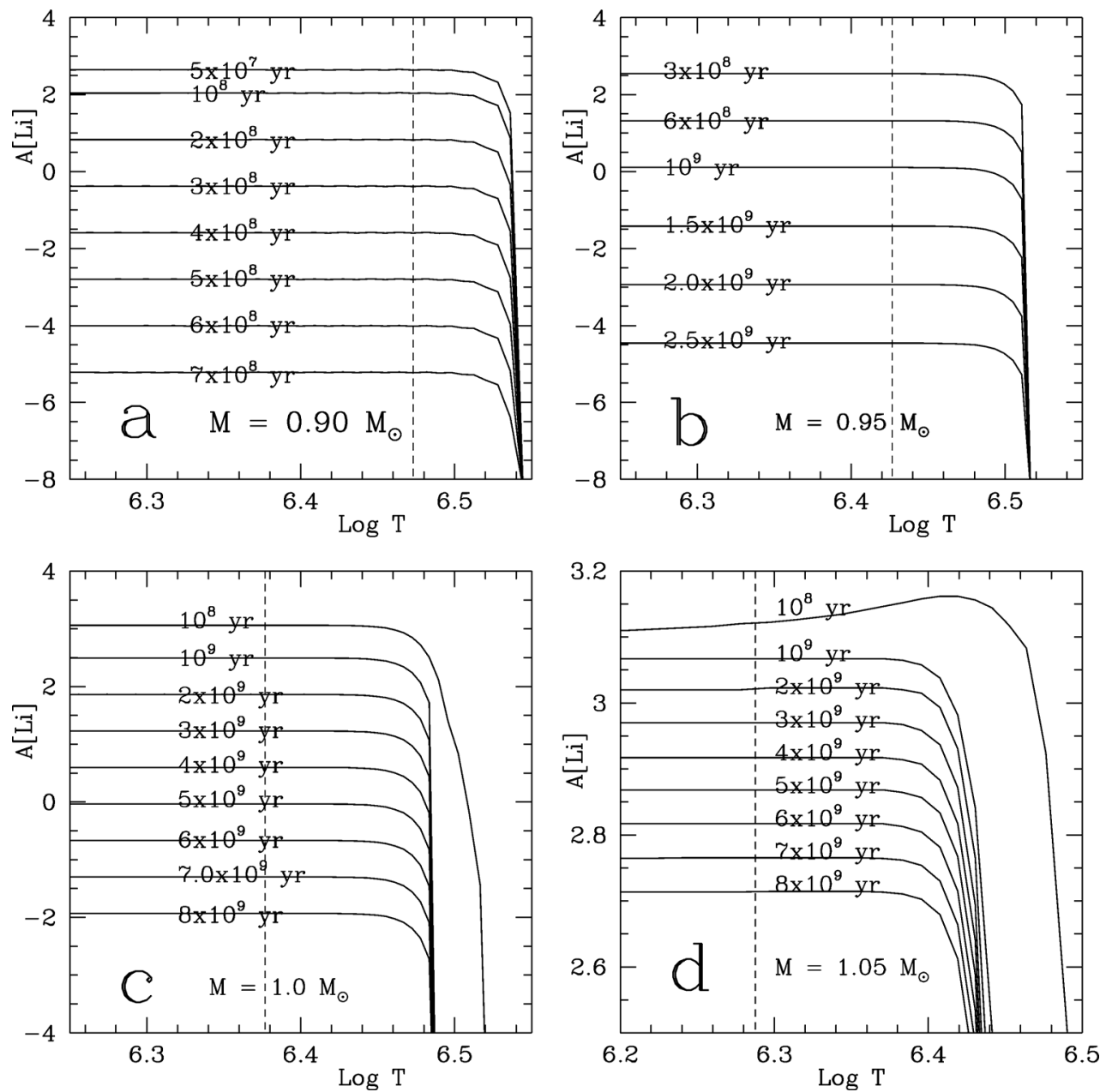

Figure 2. The Lithium abundance versus depth $(\log T)$ and age (labeled on the curves) for main sequence stars. The dashed vertical line indicates the location of the lower convective boundary. Evolution is not considered in the calculations. a). $\mathrm{M}=0.90 \mathrm{M}_{\odot}$; b). $\left.\mathrm{M}=0.95 \mathrm{M}_{\odot}, \mathrm{c}\right) . \mathrm{M}=1.0 \mathrm{M}_{\odot}$ and $\mathrm{d}) . \mathrm{M}=1.05 \mathrm{M}_{\odot}$.

\section{Acknowledgements}

The Chinese National Natural Science Foundation (CNNSF) is acknowledged for support through grants 10778719, 10773029, 10573022 and 10333060.

\section{References}

Böhm-Vitense, E., 1958, Astrophysik. 46, 108

Bressan, A., Bertelli, G. \& Chiosi, C., 1981, A\& $A, 102,25$

Canuto, V. M., 1993, ApJ, 416, 331

Canuto, V. M. \& Dubovikov, M., 1998, ApJ, 493, 834

Chiosi, C. \& Summa, C., 1970, ApSS, 8, 478

Deng, L. \& Xiong, D. R., 2006, ApJ, 643, 426

Deng, L. \& Xiong, D. R., 2008, MNRAS, 386, 1979

Grossman, S. A., Narayan, R., \& Arnett, D., 1993, ApJ, 407, 284

Kuhfuss, R., 1986, A\&̈A, 160, 116 
Kupka, F., 2003, in Modelling of Stellar Atmosphere, IAU Symp. 210, eds N. Piskunov, W. W. Weiss \& D. G. Gray, p.143 (Pub. ASP)

Leighton, R. B., Neyes, R. W., \& Simon, G. W., 1962, ApJ, 135, 474

Maeder, A., 1975, A\&BA, 40, 303

Monteiro, M. J. P. F. G., Christensen-Dalsgaard, J., \& Thompson, M. J., 2000, MNRAS, 316, 165

Salucci, G., Bertello, L., Gavallini, F. Ceppatelli, G. et al., 2004, MNRAS, 285, 322

Schwarzschild, M. \& Harm, R., 1958, ApJ, 128,348

Spiegel, E. A., 1963, ApJ, 138, 216

Stothers, R., 1970, MNRAS, 151, 65

Ulrich, R. K., 1970, ApSS, 7, 183

Xiong, D. R., 1977, AcASn, 18, 86

Xiong, D. R., 1981, SciSn, 23, 1139

Xiong, D. R., 1986, $A \mathscr{E} A, 167,239$

Xiong, D. R., 1989, $A \mathscr{E} A, 213,176$

\section{Discussion}

LUDWIG: In case of overshooting at the upper boundary: in case of the Sun this means overshooting into optically thin regions. How do you handle the radiative transfer in this situation

DEnG: For the case of the Sun, the overshooting under consideration does not enter the atmosphere (optically thin regions) therefore it is not an issue here.

Canuto: How did you model the third-order moments?

DENG: In Xiong's theory of convection, the correlation are truncated at the second order, the 3rd order moments are dealt with by a gradient type approximation.

Christensen-Dalsgand: This is a comment: The helioseismic constraint limits the sharpness of the transition in the sound-speed gradient at the base of the convection zone as found by, e.g. in the model of Zahn. The observations in fact required a smooth transition than in models without overshoot. Your model, with subadiabtic gradient in the convection zone, may satisfy that. We should look at the temperature structure in your models. Also, compare with the model of Rempel.

DENG: Indeed, we should discuss this later, and we are really interested in doing some work together especially on inversion using our models.

LANGER: This is a question actually for Canuto: What is the key difference between Canuto's and Deng/Xiong's model for overshooting?

Canuto: The Deng-Xiong's model assumes a specific form for the 3rd-order moment $F_{K E}$ that represents the flux of $T_{K E}$. It was shown (many years ago) that the DGA (down-gradient-approximation) used by Deng-Xiong may be very far from the true form of $F_{K E}$. I have therefore decided to avoid entirely any modelling of $F_{K E}$. Rather I derived a differential equation for $F_{K E}$ which contains only 2nd-order moments. The equation for $F_{K E}$ is now being solved. 